\title{
Téoros
}

Revue de recherche en tourisme

\section{Tourisme national et tourisme international au Maroc : une réelle complémentarité}

\section{Mohamed Berriane}

Volume 13, numéro 2, été 1994

Regards sur le tourisme dans les pays en développement

URI : https://id.erudit.org/iderudit/1077761ar

DOI : https://doi.org/10.7202/1077761ar

Aller au sommaire du numéro

Éditeur(s)

Université du Québec à Montréal

ISSN

0712-8657 (imprimé)

1923-2705 (numérique)

Découvrir la revue

Citer cet article

Berriane, M. (1994). Tourisme national et tourisme international au Maroc : une réelle complémentarité. Téoros, 13(2), 29-33.

https://doi.org/10.7202/1077761ar d'utilisation que vous pouvez consulter en ligne. 


\title{
Tourisme national et tourisme international au Maroc : une réelle complémentarité
}

\author{
Mohamed Berriane*
}

Àcôté d'un tourisme international qui s'est imposé au Maroc au débutdes années 1970 marquant, dans certaines régions, l'espace et les hommes, on ne peut plus ignorer aujourd'hui la montée d'un tourisme national qui, existant déjà sous des formes traditionnelles, adopte des formes modernes et revêt un caractère massif surtout au cours des mois d'été.

Cette ascension qui s'impose à l'observateur à travers l'afflux croissant d'année en année des vacanciers marocains quittant essentiellement les villes pour se diriger vers des destinations touristiques diverses, avec une préférence pour les sites balnéaires, ne se traduit que partiellement dans les statistiques officielles, n'a attiré que tardivement l'attention des chercheurs et ne bénéficie que de quel ques mesures partielles de la part des décideurs.

En effet, s'il a été longtemps admis - parmi leséconomistes en particulier - que les pays du Tiers-Monde étaient incapables d'engendrer un mouvement touristique notable, les chercheurs nient de moins en moins, aujourd'hui, l'existence d'un tourisme propre à ces sociétés. Mais celui-ci est rarement reconnu comme un élément important dans les relations de ces sociétés avec leursespaces ${ }^{(1)}$. Lesquelquesanalysesayant abordé le thème du tourisme des nationaux danslespaysen développement ${ }^{(2)}$ se fonten outre remarquer par l'absence totale de toute réflexion sur les articulations entre le tourisme national et le tourisme international. Or, ces articulations et rapports sont réels et une réflexion relative au tourisme et son évolution dans un pays comme le Marocne peut plusignorer la dimension interne.

Pour le Maroc, on peut rappeler ici que les nationaux consomment selon les années jusqu'a $20 \%$ des nuitées hôtelières enregistrées dans les établissements classés et l'ampleur des déplacements est aujourd'hui

Monsieur Mohamed Berriane est protesseur de gégraphie a la Faculté des Lettres et des Sciences Humaines âl'Université MohammedV, Rabat. telle qu'un Marocain citadin sur trois part chaque été en vacances, que le trafic-voyageur supplémentaire d'été enregistre des écarts par rapport à la moyenne compris entre $27 \%$ et $42 \%$ et que certaines petites villes et villes moyennes d'accueil voient leurs populations doubler au cours du mois d'été.

Ceci dit, il ne s'agit pas, ici, de s'attarder à démontrer l'existence de ce tourisme national au Maroc ou de se livrer à une sorte de plaidoyer en sa faveur ${ }^{(9)}$. Le but recherchéici se limite aux seules articulations existant entre le tourisme des nationaux et celui des visiteurs étrangers pour en tirer les enseignements nécessaires à une nouvelle politique plus globale du tour isme au Maroc. En s'appuyant sur une analyse comparative des deux types de tourismes, le national et l'international, on peut souligner le rôle du tourisme des nationaux comme complément du tourisme international. Son développement constitue, en fin de compte, un élément essentiel dans une nouvelle stratégie de la politique touristique du pays.

Pour ce faire, nous allons axer la démonstration sur trois points :

1) La complémentarité existant entre les deux tourismes sur le plan spatial.

2) La complémentarité existant entreles deux tourismes dans le temps.

3) Le rôle économique du tourisme intérieur au niveau des investissements et au niveau des économies locales.

Une condusion reprendra les divers aspects de la complexité des articulations existant entre le tourisme international et le tourisme national.

\section{La complémentarité dans l'espace}

La répartition spatiale des nuitées hôtelières du tourisme national est sensiblement différente de celle des nuitées du tourisme international

La comparaison entre la répartition géographique des nuitées hôtelières de la clientèle nationale d'une part et celle des nuitées revenant aux touristes étrangers d'autre part est riche d'enseignements.

Il faut souligner en premier lieu $h$ forte ancentration spatiale des nuités ausi bien marocaines qu'étrangères. Les Marocains concentrentl'essentiel de leursnuitées $(62,6$ $\%$ ) dans 5 provinces (sur 32) qui sont, par ordre décroissant, Casablanca, Agadir, Marrakech, Tanger et Tétouan. Mais les séjours du tourisme international sont encore plus concentrés: deuxvilles seulement (Agadir et Marrakech) regroupent plus de $50 \%$ du total des nuitées. Cette relative diffusion du tourisme national aura des conséquences appréciables sur les retombées de cette activité.

Les lieux de séjour qui reçoivent l'essentiel des flux ne sont pas tout à fait les mêmes pour les deuxclientèles. Agadir, Marrakech et Tanger sont, certes, les villes les plus fréquentées aussi bien par les nationaux que par les visiteurs étrangers. Mais, alors que pour ces derniers c'est Agadir qui arrive en tête, suivie de Marrakech, les Marocains privilégient Casablanca et ajoutent une quatrième ville, Tétouan.

Au niveau des ensembles régionaux, des nuances entre les deux types de fréquentations sont à relever. Chez les Marocains, l'Atlantique Nord (entre Kénitra et El Jadida), suividela Méditerranée (y compris la ville du détroit), de l'Atlantique Sud (Agadir, Essaouira et Safi), des régions de Marrakech et de Fes-Meknès sont les régions les plus recherchées. Les touristes étrangers se dirigent, quant à eux, de préférence vers l'Atlantique Sud, la Méditerranée et Marrakech (tableau 1). À noter également l'importance relative pour les Marocains du Moyen Atlas et de l'Oriental, régions qui, inversement, sont peu touchées par les flux du tourisme international.

Mais cette géographie des séjours touristiques ne concerne que les séjours ayant lieu dansleshôtels, lesvillages devacances et les résidences touristiques homologués. Lorsque l'on prend en compte les autres 
modes d'hébergement, la situation change sensiblement.

Il apparait, en dernière analyse, que les régions les plus recherchées par les touristes nationaux soulignent la spécificité du tourisme marocain. Un partage de lespace touristique semble s'instaurer entre d'une part les vacanciers nationaux et d'autre part les visiteurs étrangers. Les premiers se réservent (ou récupèrent) de plus en plus le Nord du poys (Tanger et la cote méditerranéenne), lave athantigue compris entre Asilab et Fl fadida, et secondairement, Le Moyen Atlas tabulaire. Les seconds iwvetissent aspant tout les destinations méridionales anec Agadir, Marrakecbetle Sud intérieur.

Au sein de l'ensemble des lieux de séjours des touristes marocains, les villes petites et moyennes occupent une place remarquable. En leur ajoutant les stations isolées des grands centres urbains, ces villes ont totaliséd'après les déclarations de nos enquêtés $51,8 \%$ des séjours de l'été 1985 . En effet, alors que le touriste international, même lorsqu'il éprouve le besoin de s'isoler, se dirige avant tout vers les grandes villes (Agadir, Marrakech, Tanger), les petites villes, les villes moyennes et divers petits autres centres sont les receptades d'une partie non négligeable des flux du tourisne interne qui émanent principalement des grandes agglomérations. La raison essentielle avancée pour lechoix de ces pointsde chutey est le coût relativement bas de la vie, comparé à celui qui est enregistré à Tanger et, plus encore, à Agadir.

C'est là un premier aspect de cette complémentarité existant entre les pratiquestouristiquesdes Marocains etcellesde la clientèle internationale. Mais il existe une deuxième complémentarité, encore plus importante pour la commercialisation du produit marocain, et qui se rapporteà la fréquentation touristique dans le temps.

\section{La complémentarité dans le temps}

Alors que le tourisme des nationaux est surtout estival, celui des visiteurs étrangers est de plus en plus marqué par des pointes printanières et hivernales.

\section{Un tourisme surtout estival}

Les mois d'été apparaissent comme la saison privilégiée des départs des Marocains en vacances ou en voyages touristiques. Ceci est souligné aussi bien par les déclara-

TABLEAU 1

Répartition des nuitées hôtelières des touristes national et international (moyenne des annees 1981 i 1986 )

\begin{tabular}{lcrrr}
\hline \multirow{2}{*}{ Régions } & Nationaux & & \multicolumn{2}{c}{ Touristes ctrangers } \\
\cline { 2 - 5 } & Effectifs & $\%$ & Effectifs & $\%$ \\
\hline Atlantique Nord & 443921 & 26,7 & 190419 & 2,3 \\
Méditerranće & 411600 & 24,8 & 1343978 & 16,6 \\
Atlantique Sud & 243131 & 14,6 & 3243290 & 40,0 \\
Marrakech & 205611 & 12,4 & 1212550 & 15,0 \\
Fès - Mekness & 187600 & 11,3 & 453162 & 5,6 \\
Oriental & 59839 & 3,6 & 43015 & 0,5 \\
Moyen Atlas & 43977 & 2,6 & 100464 & 1,2 \\
Sud - intérieur & 13003 & 0,8 & 258430 & 15,3 \\
Reste du pays & 52210 & 3,1 & 1242662 & 100,0 \\
\hline TOTAL & 1660892 & 100,0 & 8081970 & \\
\end{tabular}

tions recueillies lors de nos deux enquêtes principales que par la répartition mensuelle des nuitées hôtelières. L'observation directe, enfin, des modifications affectent, au cours de l'été, le rythme de la vie quotidienne de certaines villes confirme cette constatation.

Si $26 \%$ des ménages de notre échantillon (Enquête, Berriane) partent au cours des vacances scolaires d'hiver et $28 \%$ au cours de celles du printemps, l'été reste la saison par excellence des grands départs: plus de $30 \%$ du total des ménages enquêtés dans les villes de départ si nous ne considérons que les départs en famille mais $42 \%$ si nous prenons en compte tous les ménages qui ont enregistréle déplacement $d^{\text {'un }}$ de leurs membres.

Concentrés au cours de la saison estivale, à l'échelle de l'année, les départs le sont aussj à l'échelle du mois. Sur l'ensemble des départs déclarés pour l'été 1985 (enquête lieuxdedépart), $32,6 \%$ ont eu lieu au cours des 10 premiers jours du mois de juillet, 8,6 $\%$ au cours de la seule journée du 15 juillet et $18,5 \%$ la première semaine du mois d'août.

\section{Les causes de cette} concentration estivale Cette concentration renoue tout d'abord avec la tradition

Rappelons, en effet, que la belle saison est celle qui est traditionnellement l'occasion des fêtes individuelles et collectives. Les moussems se concentrent au cours de l'été, saison propice - à la fois pour des raisons climatiques et śconomiques - à toutes les manifestations qu'ils comportent. C'est au cours de la saison estivale aussi que l'aristocratie des anciennes capitales, Fès et Marrakech, a pris l'habitude - dès le début du siècle - de fuir les chaleurs torrides de ces deux villes de l'intérieur. Pour ce faire, ces failles migraient soit vers les villes côtières(El Jadida, Essaouira pourMarrakech, Tanger et Tétouan pour Fès), soit vers lat basse et moyenne montagne (vallée de l'Ourika pour Marrakech et Moyen Atlas pour Fes).

Cetteconcentration est devenue incontournable grâte au rythne qu'imiprine la vie moderne atur villes

La scolarisation - relativement généralisée dans les villes - a hérité du système scolaire français les traditionnelles grandes vacances estivales. Celles-ci se situent officiellement entre le ler juillet et le 15 septembre et rythment désormais la vie de la majorité des ménages. Le travail salarial et les emplois dans la fonction publique accusent cette nouvelle division de l'année. Le congé payé, d'une durée d'un mois, est généralement pris au cours de l'été à l'occasion des vacances scolaires des enfants. Ces congés administratifs expliquent la coincidence fréquente entre les principales vagues de départs et le ler, le 15 ou le 30 des deux mois d'été. Hérités du système occi- 
dental, les congés annuels demeurent concentrés dans le temps au Maroc.

Les raisons d'ordre socio-économique sont ausi décisives que les précédentes

L'examen des taux de départ au cours des différentes saisons et selon les groupes sociaux introduit en effet quelques différences de comportements entre ces groupes. Seules les classes supérieures ont, non seulement des départsestivaux fréquents, mais aussi des taux de départ appréciables au printemps et en hiver. Tous les autres groupes - y compris les classes moyennes partent peu le reste de l'année. La contrainte semble ici d'ordre économique, la prédominance d'un seul départ au cours de l'année (situé de préférence en plein été) est donc en relation étroite avec les possibilités financières des ménages. L'idée d'étalement des vacances - à l'ordre du jour dans le sociétés technologiquement et économiquement avancées - est totalement absente au Maroc.

Ces départs estivaux des nationaux sont à opposer à des arrivées des touristes étrangers plus étalées dans le temps

La fréquentation des hôtels par le tourisme intérieur se distingue à la fois de celle des résidents étrangers et de celle des touristes internationaux. La répartition mensuelle desnuitées desrésidentsétrangersestmieux répartie au cours de l'année aveccependant une pointe printanière bien nette. Celle des nuitées du tourisme international est marquée par une saison touristique principale correspondant au printemps.

Cette brive comparaison permet de bien mettre en évidence la spécificité de la fréquentation marocaine, dansilensembleplutôt estioale ${ }^{(4)}$. Flle suggè̀re ausi lintérêt de la composante nationale pour le développentent du tourisme en général. En effet, la saison des grands départs des touristes marocains ne coincidant pas exactement avec les saisons d'arrivées mascives des visiteurs étrangers, cette demande interne nous semble entrer peu en concurrence avec le mardbé international et pourrait mêne, si elle était judicieusement utilisée et encouragée, fournir un sérieux complénent à ce marcbé. Elle pourrait ains contribuer à ré́oudre - au woins partiellement - le probleme de la saison creuse internationale, si elle était canalisée vers des périodes de l'année peu demandés par les marchands étrangers de sejourers touristiques. Certains bôteliers, notamment à Agadir, ont bien saisi cette complémentarité et ont lancé, ces dernières annéer, des campagnespromotionnelles visant la dientèle nationale.

\section{La complémentarité économique}

S'il est difficile d'évaluer l'impact exact du tourisme intérieur sur l'économie locale à cause de l'importance du secteur informel aussi bien au niveau de l'offre que de la demande, il est possible d'imaginer que ces retombées se diffusent beaucoup mieux dans les milieux d'accueil que ceux du tourisme international. Mais le premier rôle économique joué par le tourisme intérieur marocain nous semble être d'abord sa capacité à sauver des investissements lourds destinés initialement au tourisme international.

\section{Le sauvetage par la demande interne d'investissements touristiques destinés au tourisme international}

Écartelés entre les choix volontaristes de l'État quivisaient le tourisme international, d'un côté, et la demande pressante du tourisme national en résidences secondaires, de l'autre, les rivages des côtes méditerranéennes marocaines représentent un bel exemple de ces stations.

Il s'agit de stations touristiques conçues au départ grâce à l'intervention massive de l'Ettat et destinées avant tout à accueillir le tourisme international. Pour diverses raisons, ces stations ont eu des difficultés à drainer des flux importants du tourisme international et se trouvent aujourd'hui en partie ou totalement investies par le tou= risme national.

\section{La phase du tourisme international: de 1965 à 1973}

La côte méditerranéenne est choisie au milieu des années 1960 pour recevoir les premières implantations destinées au tourisme international. Fllevoit naitre unesérie de stations balnéaires à gestion et dientèle étrangères (Berriane, 1980 et 1988).

Elle bénéficie pleinement des mesures prises lors de la rédaction du Plan de développement de 1965-1967 (Berriane, 1980). Pour attirer les promoteurs privés dans la région, l'Etat supporte de lourdes charges financières.
L'effort de l'État va s'étendre aux investissements directs. Il participeau financement de $95 \%$ des lits créés au cours de cette première phase. Par le biais d'organismes financiers et de sociétés semi-publiques (Caisse de Dépôt et de Gestion, Royal Air Maroc, Office National Marocain du Tourisme), l'État est présent dansplusieurs sociétés touristiques s'occupant de l'équipement et de la gestion hôteliers (MarocTourist, Société Marocaine pour le Développement Touristique, Société Africaine du Tourisme).

C'est l'Étatégalement qui prend en charge les travaux de viabilisation et d'infrastructure en ouvrant routes principales et routes d'accès et en réalisant adduction d'eau, électricité, assainissement et communications.

Tous ces efforts aboutissent à la naissance sur la côte de Tétouan, du Sud a u Nord, des stations de Calo Negro (ClubMéditerranée et ensemblerésidentiel de la SAT), de Mdiq (un village devacances danslecentre même, le Holiday Club et le complexe MarocTourist légèrement à l'écart) et de Restinga (un complexe Maroc-Tourist et un Club Méditerranée).

Au cours de la décennie 1970 et de la prentiere moitié de la décennie suivante, la côte méditerranécnne tombe dans Foubli (1973-1983)

Très vite les Tours Opérateursse détournent de la côte méditerranéenne. Après les premières années d'euphorie, la côteméditerranćenne est vite abandonnée par les agences internationales qui se tournent vers les destinations méridionales. La nécessité de lancer sur le marché de façon régulière des destinations nouvelles, le succès de plus en plusgranddu «produithiverm, les avantages commerciaux d'une station ouverte toute l'année - comme Agadir - et l'absence d'une animation spontanée dans un environnement rural où lesétablissements touristiques sont isolés, expliquent ce changement d'attitude des Tours Opérateurs visà-visde la côte Nord au profitd'Agadir. De ce fait, les rythmes des constructions se ralentissent dès le début des années 1970 pour s'arrêter définitivement à la fin de la même décennie.

Durant la période 1973-1984, peu de réalisations de grande envergure voient le jour. Durant une quinzaine d'années, les stations de la côte tétouanaise - comme d'ailleurs celles d'Al Hoceima - confiées 
rapidement pour la plupart d'entre elles à des sociétés à gestion étrangère (Club Méditerranée) pour assurer un taux de remplissage convenable, continuent à fonctionner en vase clos sans la moindre extension ou nouvelle création.

Il faudra attendre le milieu des années 1980 pour assister à une relance effective de la région. Phénomène original et insolite, cette relance est le fait du tourisme national.

\section{L'arrivée en force du tourisme national de 1983 à nas jours}

En 1983, la société Maroc-Tourist met en vente une partie du parc de logements prévu pour accueillir le tourisme international. 50 appartements et 42 chalets sont ainsi cédés à des particuliers de nationalité marocaine. Cettedateconstitue pour nous, de ce fait, une rupture symbolique dans l'évolution du tourisme sur le littoral Nord: le remplacement progressif de la clientèle étrangère par des touristes nationaux.

La région connaît, à partir du début de la décennie 1980 , une véritable effervescence dans l'aménagement de nouveaux ensembles touristiques. Les chantiers en cours une fois terminés, l'accroissement de la capacité d'accueil offerte par la région serait de l'ordre de $148 \%$. Cette augmentation brutale du parc d'hébergement touristique s'accompagne d'importantes mutations.

\section{L'affirmation d'un nouveau type d'bébergement}

Les réalisations des stations de la première génération étaient axées sur la formule du village de vacances à gestion étrangère. Celui-ci représentait plus de $60 \%$ de la capacité totale de la région en 1978 et $43 \%$ en 1984. Aujourd'hui, un nouveau type que nous appellerons résidentiel s'affirme de plus en plus. C'est ainsi que les ensembles d'appartements, studios, chalets, bungalows et villas de différents standing qui représentaient $46,8 \%$ en 1984 , vont constituer $76,6 \%$ du total des litsà la fin des travaux en cours. Si nous ne considérons que la capacité des chantiers en cours, les ensembles résidentielsindividuels, ou semicollectifs représentent $94 \%$ des lits en cours de réalisation. La capacité additionnelle prévisible par les projets déposés maintiendra un taux assez élevé de ce type d'hébergement: $77 \%$.

Le village de vacances, établissement par excellence du touriste étranger, verra son poids relatif baisser considérablement: 16 $\%$ à la fin des travaux engagés et $8,8 \%$ en incluant les prévisions des projets en cours d'examen. L'hôtel classique, parent pauvte dès le départ, continuera à servir de support à des complexes touristiques où la résidence individuelle, collective ou semicollective est la pièce maîtresse de l'ensemble.

\section{Une meilleure diffusion des retombées} économiques sur le plan local

En essayant de comprendre le peu d'intérêt manifestépar les autorités de tutelle visà-vis du tourisme national, on peut avancer le fait que ce tourisme était supposé avoir peu d'impact sur l'économie, la société ou l'espace. Or l'une des hypothèses de cette recherche partdu fait queles déplacements touristiques internes ont des retombées non négligeables sur le milieu d'accueil.

Les touristes marocains ne disposent, certes, dans leur grande majorité que de modestes revenus, du moins par comparaison avec ceux des touristes étrangers visitant le pays. Ils sont issus pour la plupart d'entre eux des classes moyennes et des groupes sociaux sans grands moyens financiers. $\mathrm{Ce}$ serait pourtant une erreur de croire que ce type de tourisme, parce qu'il est surtout pratiqué par des familles dont le pouvoir d'achat est modeste, n'a guère d'impact sur les espaces d'accueil; bien au contraire! Exception faitedes TME, lesdéplacements touristiques nationaux ne s'accompagnent pas de rentrées de devises, mais ces déplacements contribuent à une redistribution spatiale des richesses. Ils suscitent, en effet, des transferts interrégionaux entre, d'une part les régions d'origine des touristes où se dégagent des surplus, et d'autre part les régions d'accueil qui bénéficient des dépenses occasionnées par les séjours de vacances.

Par contre on a pu démontrer que si les entrées en devises dues au tourisme international sont non négligeables pour la balance des paiements de l'État, les retombées au niveau local sont par contre très limitées(Berriane, 1978, 1980 et 1986). En schématisant, nous pouvons dire qu'exception faite des villes d'Agadir ou de Marrakech où l'influence du tourisme in- ternational sur l'économie urbaine est manifeste, les autres stations balnéaires profitent peu des retombées de cette activité. La faiblesse de l'équipement hôtelier, la stagnation de la fréquentation touristique ces dernières années et le type d'établissements (villages de vacances de grandes tailles et de gestion étrangère) expliquent cette situation. Le constat est assez inquiétant: petit nombre d'emplois créés, instabilité du personnel, faible niveau d'achatslocauxdela part du secteur hôttelier, peu de dépenses effectuées directement par les touristes eux-mêmes. Les grandes agglomérations et notamment Casablanca pompent à leur profit par l'intermédiaire de leur secteur commercial, les bénéfices tirés de cette activité économique. Les autres transferts monétaires générés par le tourisme international (location des établissements par les grandes chaînes, investissements, impôts, produits des contrats passés par les Tours Opérateurs avec les hôteliers et les agences) sont soit versés directement dans les caisses de l'État soit captés par le système bancaire installé lui aussi dans la grande ville et en dehors de la zone d'accueil.

Par opposition à cette situation, nous pensons que si levolume de la masse monétaire mobilisée par les déplacementsinternes est plus faiblequecelui qui revient au tourisme international, les apports financiers du tourisme national se diffusent dans les milieux d'accueil et y restent. En effet, le touriste marocain séjournant en location chez des particuliers, dans une résidence secondaireou en camping, entreen contact direct avec l'habitant: logement chez l'habitant, approvisionnement auprès des commerçants ou des paysans, utilisation des mêmes services que les populations locales, etc.

Ces conséquences sont particulièrement importantes pour la petite ville et la ville moyenne, qui lorsqu'elle est touristique, n'est plus ignorée par la grande ville. En recevant des populations originaires d'autres villes de la région ou de plus loin encore, la petite ville resserre ses liens avec le reste de la trame urbaine; son système de relations, notamment avec les grandes villes, principaux points d'émission de touristes, s'étoffe et est parcouru par des fluxplus intenses.

Cette conclusion n'est pas un plaidoyer pour le développement du tourisme national au dépens du tourisme international. 
Ce dernier joue un rôle déterminant au niveau des financesnationales tout en ayant, commed'autres activités économiques, des répercussions parfois négatives sur l'aménagement du territoire (déséquilibres régionaux) et sur leséconomies et les sociétés locales (déstructuration, conflits). De ce fait, si sur le plan socio-spatial, il arrive que les deux types de tourismes entrent en conflit, sur le plan économique, ils peuvent se compléter harmonieusement.

\section{Conclusion}

En conclusion, nous pouvons dire qu'il est temps de changer d'attitude vis-à-vis du tourisme interne au Maroc. En effet, examinées en termes de conflit ou de complémentarité, les relations tourisme nationa $1 /$ tourisme international se révèlent de nature assez complexe.

Le tourismeinternational qui se dirige vers le Maroc entre obligatoirement en contact avec le tourisme national. Nous n'avons retenu que trois axes (à savoir les complémentaritésspatiales, temporelleset économiques) mais les articulations sont nombreuses et réelles.

Le tourisme international peut avoir tout d'ahord un rôle d'entraînement. Il diffuse des habitudes et des pratiques touristiques exogènes tout en incitant parfois des groupes et individus au départ en voyage touristique. Mais son influencela plus voyante et la plus géographique se trouve ailleurs. C'est, nous semble-t-il, son rôle à lancer promouvoir et valoriser de manière indirecte certaines destinations auprès des touristes nationaux qui a le plus de conséquences sur le plan géographique. Le succès sur le plan interne de villes ou stations comme Marrakech, Agadir, Al Hoceima et même Tanger, doit beaucoup à l'audience internationale de ces destinations. La mise en placed'une infrastructure d'accueil homologuéedestinée initialement ù répondre à la demande internationale incite aussi la clientèle marocaine à se diriger vers les lieux ayant reçu ces infrastructures. Ailleurs, la fréquentation de certains sites, non équipés, par de jeunes touristes européens itinérants, pratiquant le camping, attire égalementles jeunes marocains étudiants, lycéens ou autres.

L'un des effets les plus sensibles du tourisme international sur les espaces qu'il fréquentemassivement, estl'augmentation du coût de la vie. Il peut, de ce fait, avoir comme conséquence directe l'élimination des catégories sociales moyennes et démunies des destinations qu'il investit le plus. Mais en même temps, la fréquentation privilégiéed'une station par le tourisme international, peut valoriser celle-ci aux yeux des catégories sociales supérieures du pays d'accueil: de nombreux ménages des classes supérieures de la ville de Marrakech ont progressivement abandonné El Jadida, destination classique de cetteville, au profit d'Agadir, principal pôle marocain du tourisme international.

Le lancement d'une station touristique moderne destinée au tourisme international se fait parfois au détriment des estivants marocains qui fréquentent le plus souvent en campeurs, des sites vierges encore disponibles. Cessites découverts par les jeunes, les TME, les classes moyennesetles groupes transitionnels, une fois convoités par les aménageurs ou les investisseurs doivent être abandonnés par les premiers utilisateurs. Le tourisme international apparaît dans ces cas comme un redoutable concurrent du tourisme national. Mais il arrive aussi que des structures d'accueil, programmées pour répondre aux besoins des visiteurs étrangers, soient récupérées par les estivants nationaux. Plus encore, ces installations initiales servent de points d'appui à l'urbanisation dont profite essentiellement la clientèle interne.

La complémentarité entre les deux tourismes apparaît surtout au niveau de la saisonnalité. Les départs des Marocains étant concentrés en été, ils ren trent peu en conflit avec les arrivées des visiteurs étrangers. Ces derniers ont tendance à étaler leurs séjours au cours de toute l'année, les pointes printanières devenant de plus en plus marquées. Les hôteliers ont d'ailleurs saisi l'intérêt de cette distorsion temporelle entre les deux tourismes, pour mener des campagnes, certes encore timides, auprès de la clientèle interne. C'est ainsi que des prix et des formules de séjours familiaux de basse saison sont proposés à Agadir, en été, et dans le Nord en hiver et au printemps.

Nous nous limitons, ici, à quelques conséquencesd'ordrespatial et commercial, mais les relations entre les deux tourismes peuvent être riches en implications sociales et culturelles. Des études pluridisciplinaires aboutiraient sûrement à d'intéressants résultats aidant à la compréhension de l'évolution des sociétés réceptrices du tourismeinternational et à la conception d'une meilleure politique touristique globale où chacune des deux dimensions - la dimension interne et la dimension internationale - aurait sa place. $f$

\section{NOTES ET RÉFÉRENCES}

(1) Voir a ce propos notre article Le tourisme des nstiona Lx au Maroc iune nouvelle approche du tourisme dans les pays en développement), An= nales de géographle, no570, 1993, pp. 131-161.

(2) Voir, en particulier, l'importante production des géographes allemands a propos du tourisme intérieur dans les pays du Moyen-Orient.

(3) Un ouvrage repienant toute cette problemstique vient d'ètre publié en mai dernier: M. Berriane, Tourisme national et migrations de loisirs au Maroc, étude góographique, Publications de la Faculté des Lettres et des Sciences Humaines de Rabat.

(4) Cecaractère estival est d'autant plus remarquable que les années 1581 et 1982 ont vu coincider le Ramadan - mais du jeâne qui enregistre généralement peu de départs - avec le mois de juillet.

Berriane, M., L'espace touristique maracain, URBAMA, Fascicule de recherche no $7,1980$.

Berriane, M., Le tounsme et la petite et moyenne vile au. Marcc, Lapetite et moyenne ville dans le Monde Arabe, URBAMA, Fascicule de recherche no 16 , 1986.

Berriane, M., Entwicklung und Struktur des Binnentourismus in Marokko, Institu für Tounismus, Freie Universität Berlin, 1988.

Berriane, M., La sociéte marocaine et les laisirs, Grande encyclopédie du Maroc, wolume Sports et loisirs. 1988, pp. 119-129.

Berriane, M., Tourlsme intóriour et migrations de loisirs au Maroc: acculturation ou bvolution Interne?. Actes du colloque de Passau, RFA: Le Maroc, Espace et société, 1990

Berriane, M. Styles et comportements tounisticues d' une societe maghwebine ile cas du Marocl: ni tradition nimodemite, Oriont, 32 Jahrgang Nr. 1, Hambourg. 1991.

Berriane, $M_{i,}$ Tourisme national et migrations de loisirs (ótude géographique), These de doctorat d'Etat de géographie, Université de Tours, Publications de la Faculte des Lettres et des Sciences Humaines, habat, 1992, 500 pages.

Berriane, M., Le tourisme des nationaux au Maroc fune nourvelie aporoche du tourisme dans les pays en developpement!. Annales de góographle, no 570 , 1993, pp. 131-161.

ELOUARTI, A, Le changement culturel: I'b́mer gence d'une pratique touristique dans le lolsir urbain a l'interieur de la socióte marocaine (1975. 1985). Thèse de troisieme cycle de sociologie, Paris IV, 1985. 